\title{
Comparative Analysis of ANN Techniques for Predicting Channel Frequencies in Cognitive Radio
}

\author{
Imran Khan \\ Department of Telecommunication Engineering \\ Dawood University of Engineering and Technology \\ Karachi, Pakistan
}

Dr. Shaukat Wasi

Department of Computer Science

Muhammad Ali Jinnah University

Karachi, Pakistan

\author{
Adnan Waqar \\ Department of Electronic Engineering \\ Dawood University of Engineering and Technology \\ Karachi, Pakistan \\ Saima Khadim \\ Department of Telecommunication Engineering \\ Dawood University of Engineering and Technology \\ Karachi, Pakistan
}

\begin{abstract}
Demand of larger bandwidth increases the spectrum scarcity problem. By using the concepts of Cognitive radio we can achieve an efficient spectrum utilization. The cognitive radio allows the unlicensed user to share the licensed user band. To sense the accessibility of vacant channel and allocation of licensed user band is provided by Machine learning techniques because this decision need to be very fast and accurate. It is based on certain factors (such as Power, Bandwidth, antenna parameters, etc.). In this paper, we used neural network to propose this decision of resource allocation more accurately by providing bandwidth, power, antenna gain, azimuth, angle of elevation and location as a supplements factors to increase the predicting accuracy of Available channel frequencies for secondary user in particular bands. The comparative analysis is done between artificial neural network techniques to determine the maximum decision accuracy in order to design a suitable neural network structure and the system to make fast prediction for available channels. The dataset is divided in to cellular $850 \mathrm{MHZ}$ and Advanced wireless service 1900/2100 MHZ bands. In both bands, Feed Forward networks performs better as compared to Elman and Radial basis network for predicting the best available channel to accommodate the secondary user. It will considerably increase overall QoS and decrease interference, hence making Cognitive radio system reliable.
\end{abstract}

Keywords-Cognitive radio; machine learning; artificial neural network; frequencies band; feed forward neural network; Elman; Radial basis

\section{INTRODUCTION}

In recent studies, it has been witnessed that the allocated spectrum remain largely underutilized [1], while the remaining parts are heavily and sparingly occupied. It is perceived that this sort of static allocation techniques has brought about poor use of spectrum, and makes an absence of spectrum utilization for unlicensed users. Moreover, spectrum underutilization by licensed users compounds spectrum shortage. The major issue of spectrum underutilization is that licensed user doesn't utilize their assigned bandwidths capacities for many times. While unlicensed users are being famished for spectrum accessibility.
A direction for accomplishing spectrum better utilization is to equip the framework with cognitive radio capabilities. CR is considered as a smart radio which sense spectrum channel availability and adjust its operating parameters to communicate effectively with other user by avoiding interference [2]. As represented by authors in [2] in Fig. 1 that sensing and analysis provides the means for RF awareness, such as the presence/absence of the licensed users, their transmit powers and the channel conditions. Decision making and learning exploit the RF awareness for choosing actions that maximize the CR's utility, such as the data rate. These include choosing the operation frequency, transmit power, modulation, coding, etc., which are carried out in the adaptation step. Each secondary/unlicensed user (SU) can detect and access the spectrum when the spectrum is unoccupied by primary/licensed users (PUs). At the point when a PU solicitations to get to its own range, the SUs utilizing a similar range astutely ought to change to other vacant spectra to ensure the transmission of the PU and proceed with their own particular information conveyance.

There is a wide range of frequencies that needs to be scanned before assigning them to secondary user. If the prediction process is delayed it impacts QOS and systems reliability. If a fast but inaccurate decision is made than it creates interference. So there is a trade-off between time and accuracy. Required is a fast as well and accurate decision based on consideration of all factors required for cognitive sensing.

To circumvent this problem, this paper proposes dynamic spectrum allocation schemes together with ANN techniques to improve the performance of both primary and secondary networks by analyzing different factors that influence in spectrum management of cognitive radio using different machine learning techniques. The band considered in this study are cellular $850 \mathrm{MHz}$ band and Advance wireless service 1900/2100 band. Computational intelligence based RF frequency predictive models are developed. This will help the cognitive radio (CR) to avoid busy channels and also to prioritize its scanning by starting with the channels that are predicted to be free. 


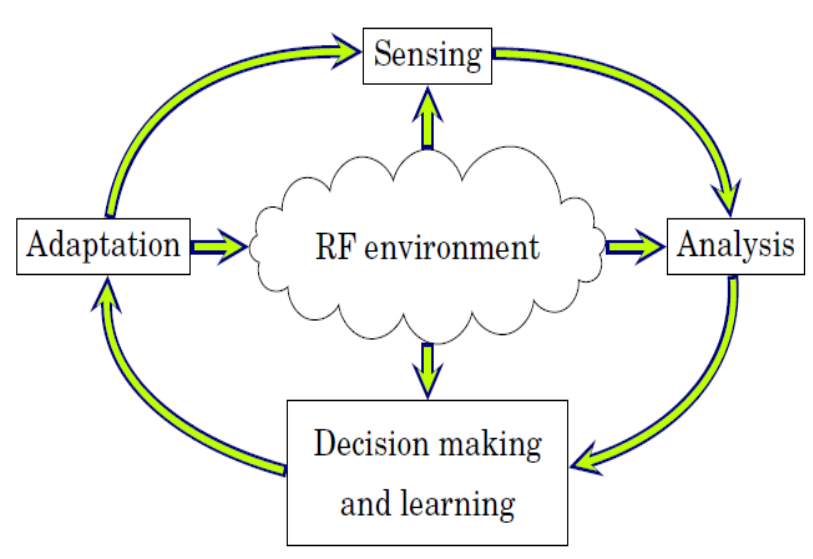

Fig. 1. Cognitive cycle of cognitive radio.

The list of all available idle frequencies and other prime factors will help the secondary user to accommodate immediately without any interruption between primary user and secondary user.

\section{RELATED WORK}

This paper presents an efficient way of spectrum resource utilization using predictive models developed by providing different parameters for prediction of real world RF frequency and bandwidth by aggregating different machine learning techniques for cognitive radio (CR) application. The prediction of factors in cognitive radio can be done through different learning algorithms. Several work has been done in the field of cognitive radio for optimization of different transmission factors. It may be possible that different types of users are present in a particular band but activity models will be definitely helpful for secondary users. Several solutions have been proposed as decision making strategies for secondary users to opportunistically access the spectrum. In this section, the most utilized techniques for learning in cognitive radio are presented such as Game theory, HMM (Hidden Markov Model), Genetic algorithms and ANN (Artificial Neural Network).

\section{A. Game Theory}

To allocate, multiple secondary users in the spectrum at the same time in cognitive radio networks, can be analyzed by game theory where each secondary user is a player of either cooperative or non-cooperative, where the secondary users exchange their information to avoid collision between them. Game theory mostly used in spectrum allocation algorithms for both cooperative and non-cooperative scenarios [3]. For dynamic spectrum sharing based on game theory for cooperative and non-cooperative scenarios was proposed in [4], [5].

\section{B. Hidden Markov Models}

For channel status prediction based on HMMs were proposed in [6], [7], where it was assumed that primary users' traffic followed a Poisson distribution with a ratio of more than $50 \%$ where primary users acquire more than $50 \%$ of the spectrum. Both schemes can be used by secondary users to predict the channel activity, in [7] the authors demonstrated its prediction accuracy but no results in these terms were provided in [6]. Moreover, the prediction accuracy of the model proposed in [7] was further evaluated in [8], [9] with synthetic data and real measurements of the $2.4 \mathrm{GHz}$ ISM and GSM1800 bands acquired by the RWTH Aachen University. The results showed that the prediction accuracy of the model proposed in [7] depends on the duty cycle of the observed channel and on the complexity of the channel occupancy by the primary user.

\section{Genetics Algorithms}

Genetic algorithms based solution provided in [10]-[13] for optimizing the parameters and channel assignment. To minimize delay and to avoid energy consumption in CR networks in [10] the authors predicts the best available channel using genetic algorithms to utilize the spectrum efficiently and improves spectrum prediction accuracy by using a combination of back propagation neural networks and genetic algorithm. For channel assignment technique genetic algorithm based solution provided in [11], where the spectrum access efficiently between secondary and primary user by adjusting operating parameters such as (the mutation rate, the population size and the number of generations). Similarly, parameter optimization in CR networks using GA also used in [12], where Bandwidth and bit error rate are adjusting parameters using mutant and crossover probability and linear scale transformation. Adaptive genetic algorithm based optimization of parameter in cognitive radio also proposed in [13] where Genetic algorithm changes its probability adaptively, Factors consider in this research are BER(Bit Error Rate), power and throughput.

\section{Artificial Neural Networks (ANN)}

Artificial Neural Networks (ANN) are massive parallel computing systems composed of large number of simple processing elements (processors) with many interconnections operating in parallel. An ANN is as neurons, which is able to keep trial knowledge and make it obtainable for usage [14]. In the context of cognitive radio, ANNs have been applied to model the activity of primary users. These models were used by secondary users to predict future primary user's activity. In a cognitive engine based on artificial neural networks was evaluated in [15]. It learnt how environmental measurements affected the performance experienced on different channels of an IEEE 802.11 wireless network. In [16], the basic purpose is to increase decision accuracy under various noisy condition and it is implemented by using Multilayer Perceptron. Neural Network that intensify or upgrade the required throughput and the performance is figure out by using three types of Neural Network through Simulation which are Perceptron Neural Network, Feed forward Neural Network and Elman Neural Network. From the Simulation results it is observed that performance of Perceptron Neural Network is better than Feed Forward Neural Network and Elman Neural Network. In [17], the authors compared four supervised learning algorithms, two from ANN, i.e. Multilayer Perceptron \& Recurrent Neural Networks, and two from Support Vector Machines (SVM), i.e. SVM with Linear and Gaussian and suggest the best learning model for accurate primary user prediction. In [18], the authors compared two NN architectures, Multilayer Perceptron( MLP) and Radial Basis Function(RBF) in term of MSE (mean square error) to predict idle and used channel availability in Cognitive radio and indicates that MLP performs more better than RBF 
for particular data. In [19], the author proposed design of cognitive radio based on Radial basis function (RBF) and genetic algorithm to adjust their parameters effectively. To adjust and adapts its operating parameters changes in the environment such as Transmitting power, data rate. In [20], the author designed an optimized ANN and SVM model to predict the RF power in UHF, TV and GSM 900 band to increase the robustness of CR applications. In [21], the authors introduces an artificial neural network based learning scheme for predicting data rate for specific radio configuration and used the algorithm for estimating the performance of data rate and throughput. In [22], the author the Combination of Cognitive Radio and Feed Forward Back Propagation both together for the replacement of Complicated Frequency allocation, from the Simulation author comparing Back Propagation (BP) Artificial Neural Network (ANN) with the original Frequency Allocation which is able to give out the accurate simulation results which means that the BP ANN gave the solution of Complicated frequency allocation and by using this method we will get accurate results of frequency allocation which also increases the Quality of Service and reduces the interference.

Literature highlights that, Ann is the most widely used algorithm for prediction of different factors in cognitive radio due to its fast adaption ability.

\section{METHODOLOGY}

Before an SU can obtain access to idle licensed spectrum it needs to proceed according to the following steps:

1) Decide which frequencies to sense.

2) Sense the chosen frequencies and decide whether the spectrum is free or not.

3) Decide whether to access or not.

The first step is managed by the spectrum sensing policy, whose task is to select the frequency band to be sensed such that persistently high data rates can be expected for the SUs. In the second step a spectrum senses the collects data from the receiver and decide whether the band is idle or occupied. In the third step the access policy decides, based on the output of the spectrum senses, whether to access the spectrum or not and which SUs get the access. For making the decision of selecting best next available channel in spectrum management of cognitive radio many transmission factors to be considered. In this research we performed a comparative analysis between different machine learning techniques of ANN by providing bandwidth, power, antenna gain, azimuth, angle of elevation and location as a supplements factors to increase the predicting accuracy in terms of MSE ( mean square error) of Available frequencies in particular bands. CR checks frequency, bandwidth, and power and antenna parameters before use of any licensed user. For spectrum decision we will be adopting a machine learning technique as an approach for Learning and Reasoning in cognitive radio. Dataset is selected that provides all factors (Frequency, power, Bandwidth, angle of elevation, azimuth, antenna gain and locations). This Dataset is trained on Artificial Neural Network using neural network toolbox of Matlab for simulations and results. The ANN has been selected for simulation are Feed Forward, Radial basis and Elman
Neural Networks. The fitness function used in this research is MSE. In this section, we will discuss about dataset and designing of different neural networks to perform these simulation.

\section{A. Dataset}

We have chosen Canadian spectrum management database available online in the website [23]. Dataset contains 33 attributes and 396163 values. Dataset Contains an extensive variety of factors related to spectrum management. The factors found over this data set comprised of many factors but we considered, transmission frequency (the center frequency in $\mathrm{MHz}$ of transmission channel of the station), transmission bandwidth (occupied bandwidth in $\mathrm{MHz}$ of transmit signal, latitude (north of the station in decimal degrees), longitude (west of the station in decimal degrees), transmit power (power fed to the transmission line in watts), transmission antenna height ( in meters), transmission antenna azimuth ( in degrees from north of the transmitter antenna), transmit antenna elevation angle, and transmit antenna gain in dbi.

We divided data set in to two parts according to two different frequencies bands offers different services.

Type -1: CELL (Cellular) 850MHZ MHZ

Type -2: AWS (Advance Wireless Service) 1700 / 2100

All categories contain ten thousand values of all above mentioned parameters in each bands.

\section{B. Feed Forward Neural Network}

The feed forward neural network is trained by first learning its input pattern and calculates the errors by backpropagation and then automatically adjusting its tap weights. It consist of input layer, hidden layer and output layer. Each hidden unit acts as a neurons and computes its activation by using activation function to modify its input values according to its transfer function. In this work, we used Tan sigmoidal or hyperbolic tangent function for hidden layer and output layer. For designing a Feed forward Network model, we have considered following values as mentioned in Table I for achieving the minimum possible mean square error.

\section{Radial Bais Neural Network}

The RBF networks consist of input vectors, RBF neurons and output vectors. The RBF neurons compares the similarities between inputs vectors and its prototype vectors. If the input matches with prototype vectors, the output of that RBF neuron is 1 otherwise is 0 . The neurons response values also known its Activation value. For designing a model for Radial Basis Function, we have considered similar values as described in Table I, for achieving the minimum possible mean square error.

TABLE I. TRAining PARAMETERS For FF NEURAL NETWORK

\begin{tabular}{|c|c|c|c|}
\hline $\begin{array}{l}\text { No. of } \\
\text { Epochs }\end{array}$ & $\begin{array}{l}\text { Learning } \\
\text { Rate }\end{array}$ & $\begin{array}{l}\text { Training } \\
\text { Function }\end{array}$ & $\begin{array}{l}\text { No. of hidden } \\
\text { Neurons }\end{array}$ \\
\hline 1000 & 0.01 & Trainlm & 20 \\
\hline
\end{tabular}




\section{Elman Neural Network}

Elman neural networks are similar to feed forward neural network but with an addition of layer (i.e. Recurrent layer) which also connected with hidden layer with tap delays. RNNs can detect trends in a signal using feedback processing. .To train a network, known output is used enabling supervised learning. To use the network to forecast, some history of the time series must exist. For designing a model for Radial Basis Function, we have considered similar values as described in Table I, for achieving the minimum possible mean square error and to compare between different ANN techniques with a same parameter to conclude which ANN performs and predict better for the given values.

\section{RESULTS AND DISCUSSION}

Our comparative analysis divided into two parts:

- In first part we analyzed the cellular 850MHZ band on the basis of frequencies on neural networks techniques (i.e. Feed Forward, Radial basis and Elman Neural Networks).

- In Second part we analyzed the AWS (Advance Wireless Service) $1700 / 2100 \mathrm{MHZ}$ band on the basis of frequencies on neural networks techniques (i.e. Feed Forward, Radial basis and Elman Neural Networks).

Our results explicitly demonstrate that which artificial neural network technique work well for predicting available frequencies and in which band in term of MSE (mean square error). In this study it is often observed that a ANN technique might be poor in predicting one combination of factors while very good in predicting another combination of factors in a particular bands.

\section{A. Analysis in Cellular 850MHZ Band}

a) Analysis of Available channels frequencies using Feed Forward Neural Network: Fig. 2 represents the block diagram of created custom feed forward neural network. In this network we have 8 inputs (i.e. Bandwidth, Latitude, Longitude, Antenna power, azimuth, gain and height. 20 hidden layers of neurons and 1 output layer to predicts frequencies by learning the targeted values.

Fig. 3 shows the performance of training of dataset in feed forward, Elman and radial basis networks. The blue line shows the training states of the data. The green line represents validation to display the performance of network generalization. The red line displays the network performance during and after training. $\mathrm{X}$-axis represents the no. of epochs and $\mathrm{Y}$ - axis represents the MSE values. The best validation performance achieved in feed forward network is 0.063667 at epochs 43 in term of MSE.

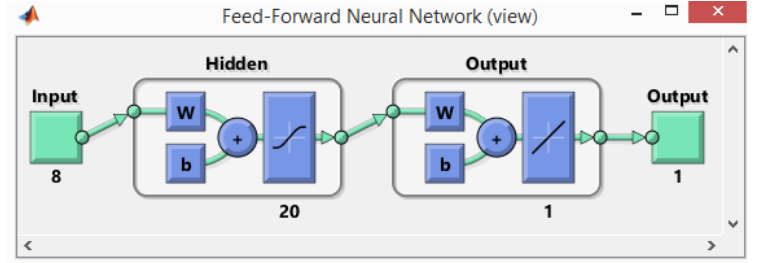

Fig. 2. Feed forward neural network.

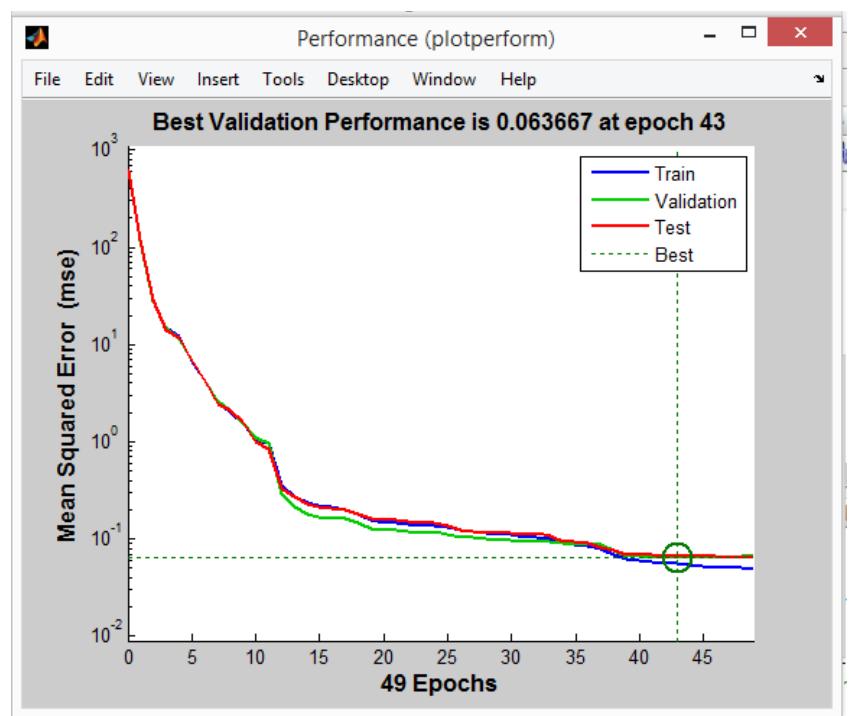

Fig. 3. Performance plot of FFNN in CELL band.

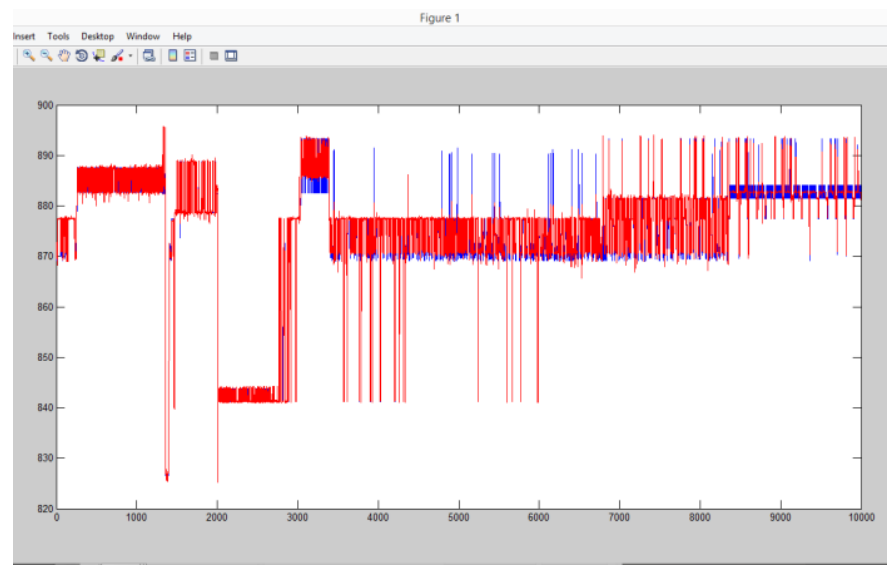

Fig. 4. Predicted and targeted values plot of FFNN in CELL band.

Fig. 4 represents the difference between targeted values and predicted values. The targeted values represent with blue line and predicted values represents with red line. $\mathrm{X}$-axis represented the no. of samples and Y-axis represents the frequencies ranges values.

b) Analysis of Available channels frequencies using Elman Neural Network: Fig. 5 represents the block diagram of created custom elman neural network. In this network we have similar inputs, hidden layers of neurons and output layer as in FFN networks to predicts frequencies by learning the targeted values.

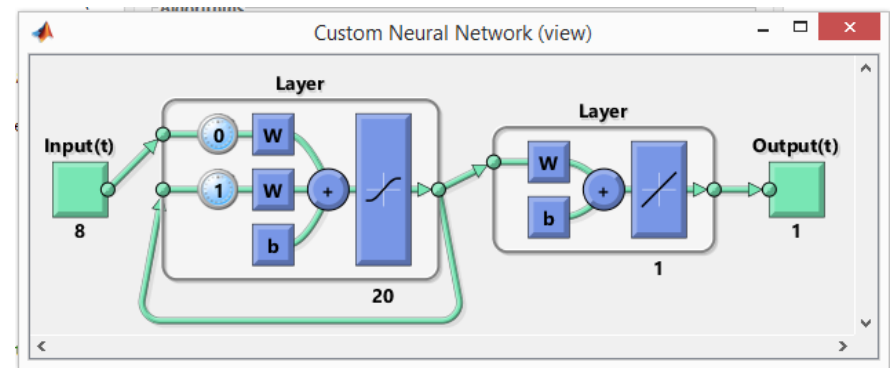

Fig. 5. Elman neural network. 


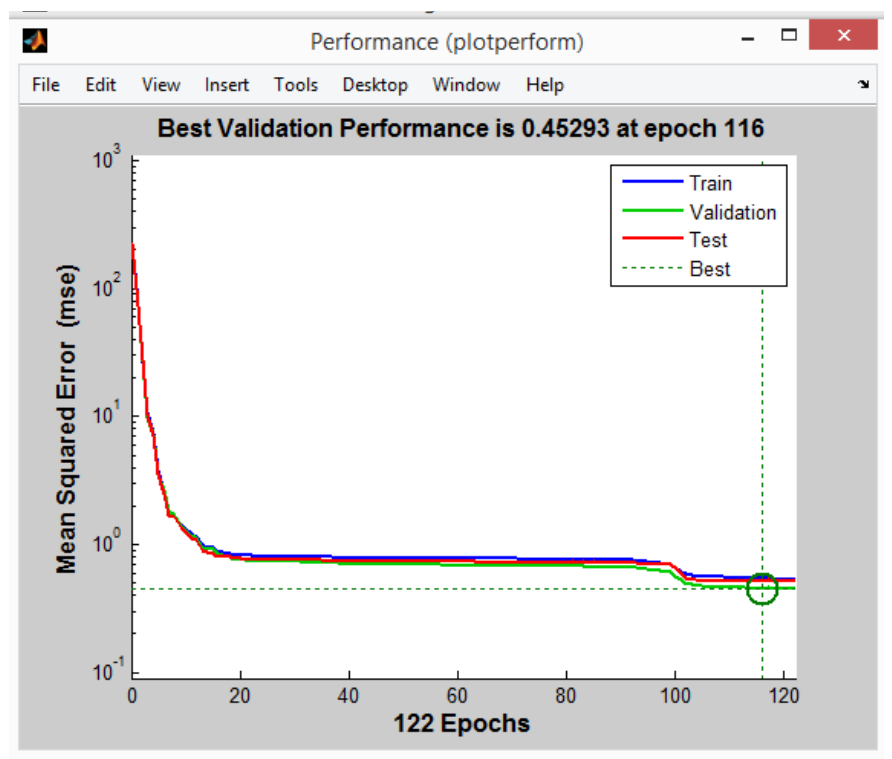

Fig. 6. Performance plot of Elman in CELL band.

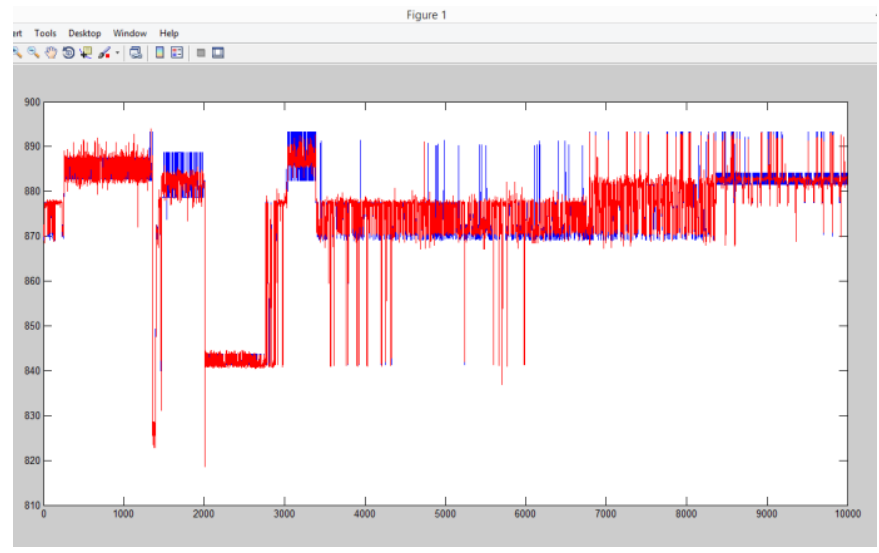

Fig. 7. Predicted and targeted values plot for Elman NN in CELL band.

Fig. 6 represents the performance plot. The best validation performance achieved in Elman Neural network is 0.45293 at epochs 116 in term of MSE and Fig. 7 represents the difference between targeted values and predicted values.

c) Analysis of Available channels frequencies using Radial Basis Neural Network: Fig. 8 represents the block diagram of created custom radial basis neural network. In this network we have similar inputs but with 1000 hidden layers of neurons because in radial basis neural networks number of epochs are equal to the number of neurons. One output layer which predicts frequencies by learning the targeted values.

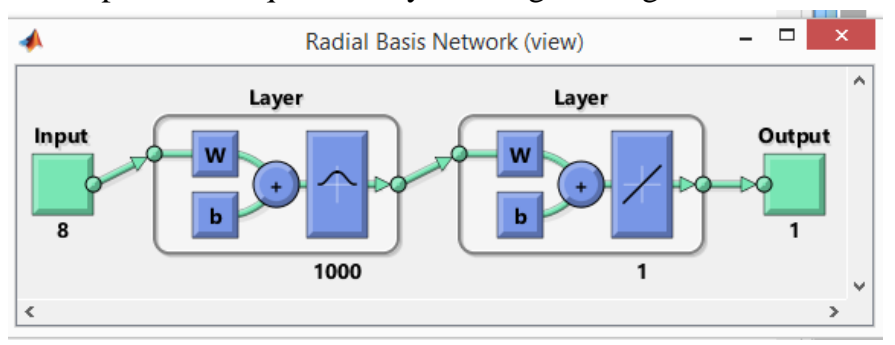

Fig. 8. Radial basis neural network.

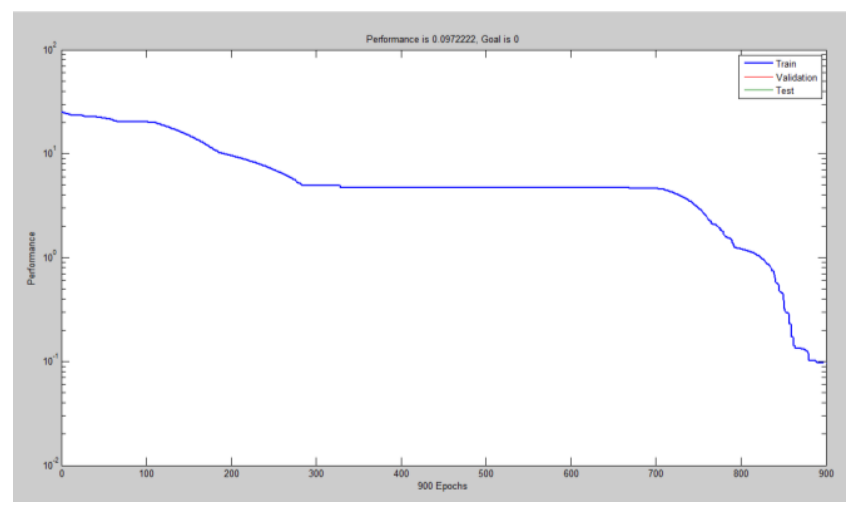

Fig. 9. Performance plot of Radial Basis in CELL band.

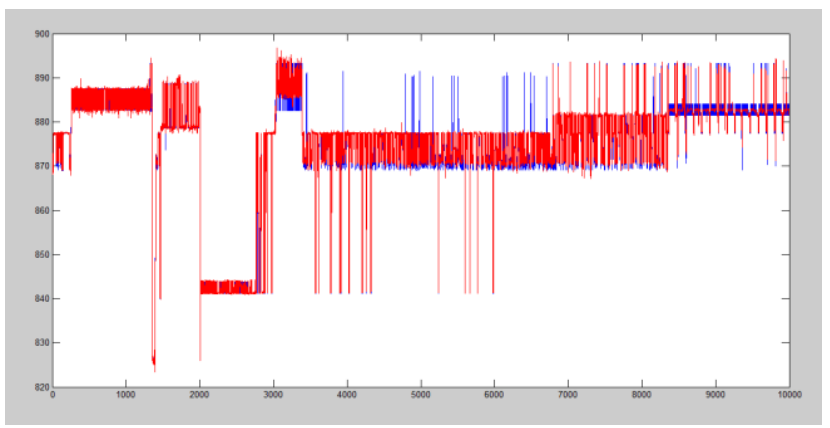

Fig. 10. Predicted and targeted values plot for Radial basis in CELL band.

Fig. 9 represents the performance plot. The best validation performance achieved in Radial basis neural network is 0.097222 and Fig. 10 represents the difference between targeted values and predicted values.

\section{B. Analysis in AWS (Advance Wireless Service) $1700 / 2100$ MHZ band}

a) Analysis of Available channels frequencies using Feed Forward Neural Network: For prediction of frequencies in AWS band same Feed Forward Neural Network model is used as shown in Fig. 2. The best validation performance achieved is 0.2186 at epochs 134 in term of MSE as shown in Fig. 11 and 12 represent the difference between targeted values and predicted values.

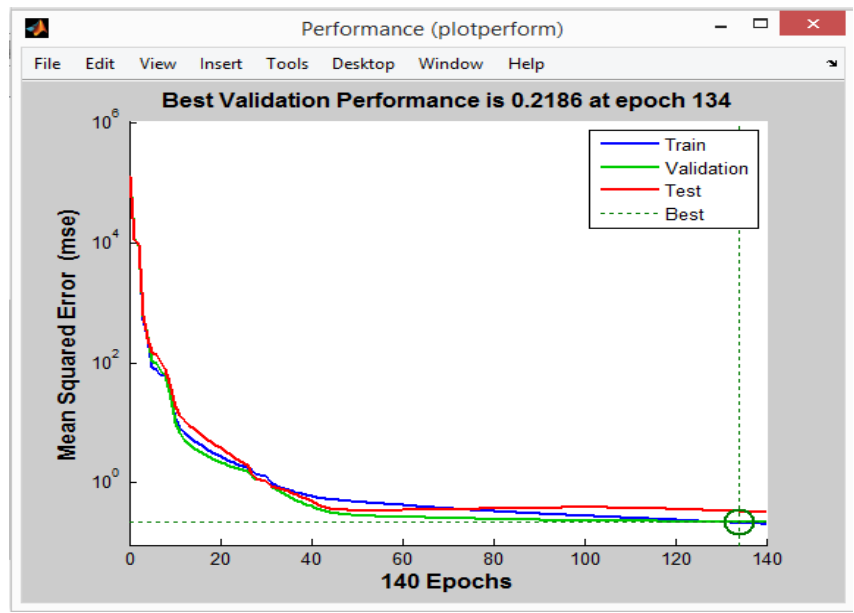

Fig. 11. Performance plot of FFNN in AWS band. 


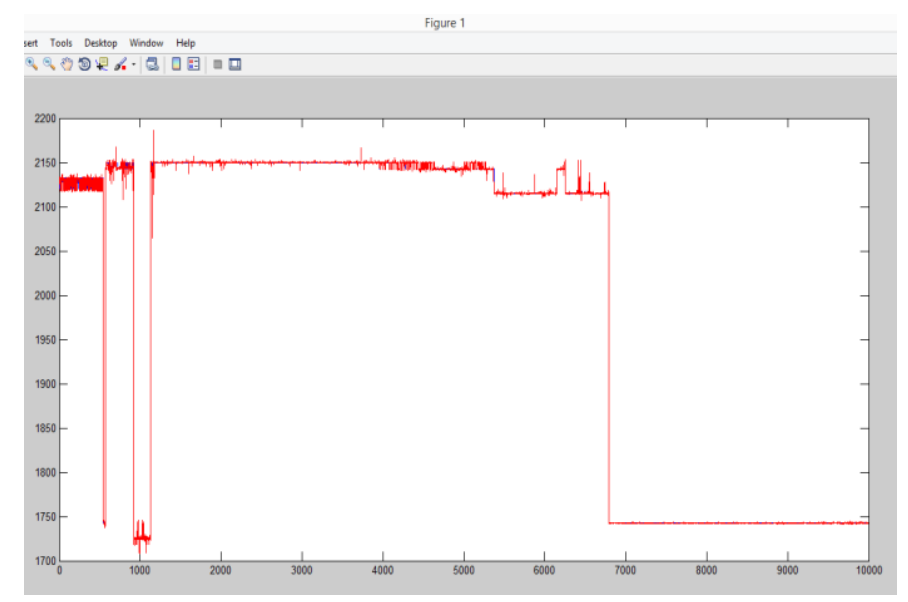

Fig. 12. Predicted and targeted values plot FFNN in AWS band.

b) Analysis of Available channels frequencies using Elman Neural Network: For prediction of frequencies in this band same Elman Neural Network model is used as shown in Fig. 5. The best validation performance achieved is 0.41012 at epochs 171 in term of MSE as shown in Fig. 13 and 14 represent the difference between targeted values and predicted values.

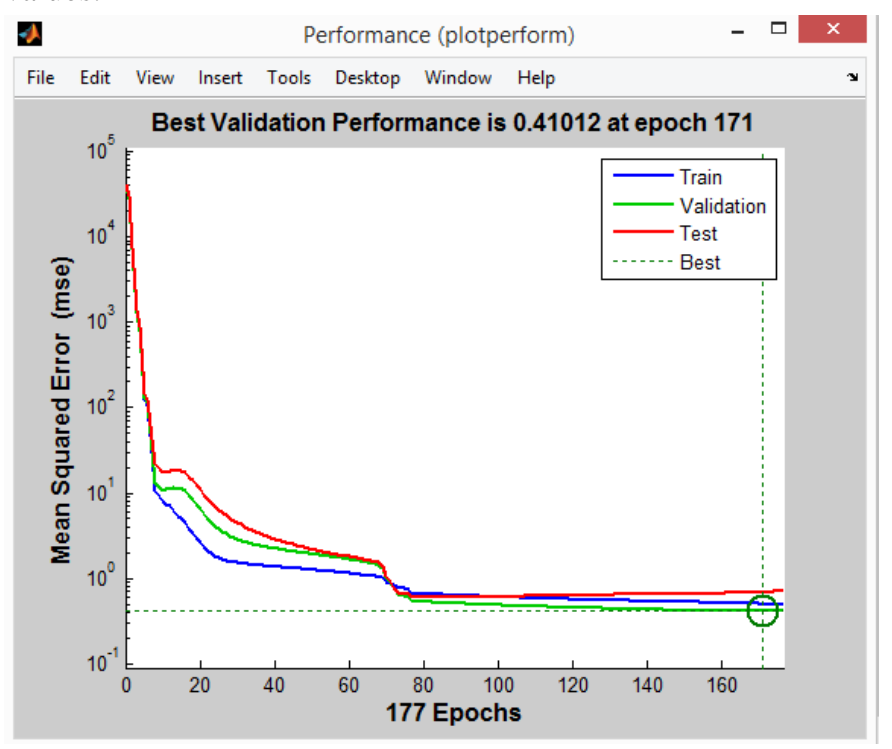

Fig. 13. Performance plot of Elman NN in AWS band.

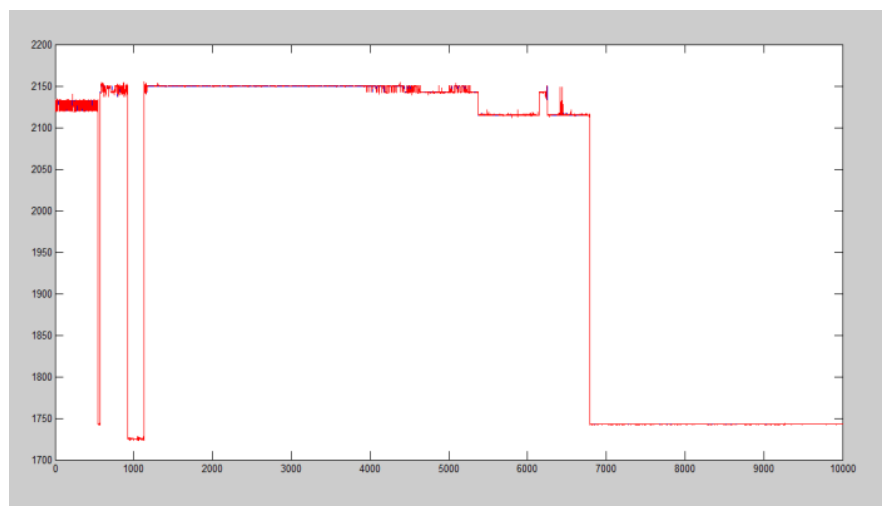

Fig. 14. Predicted and targeted values plot Elman NN in AWS band.

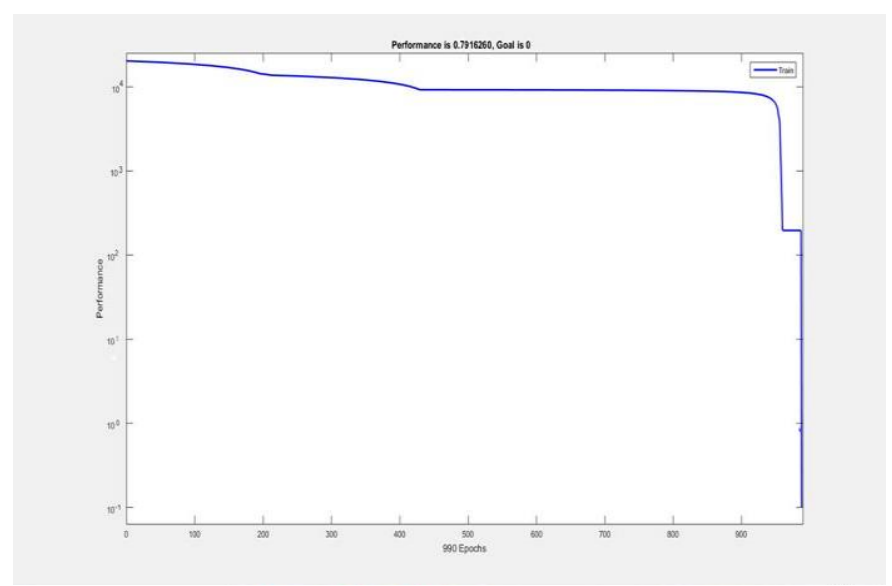

Fig. 15. Performance plot of radial basis NN in AWS band.

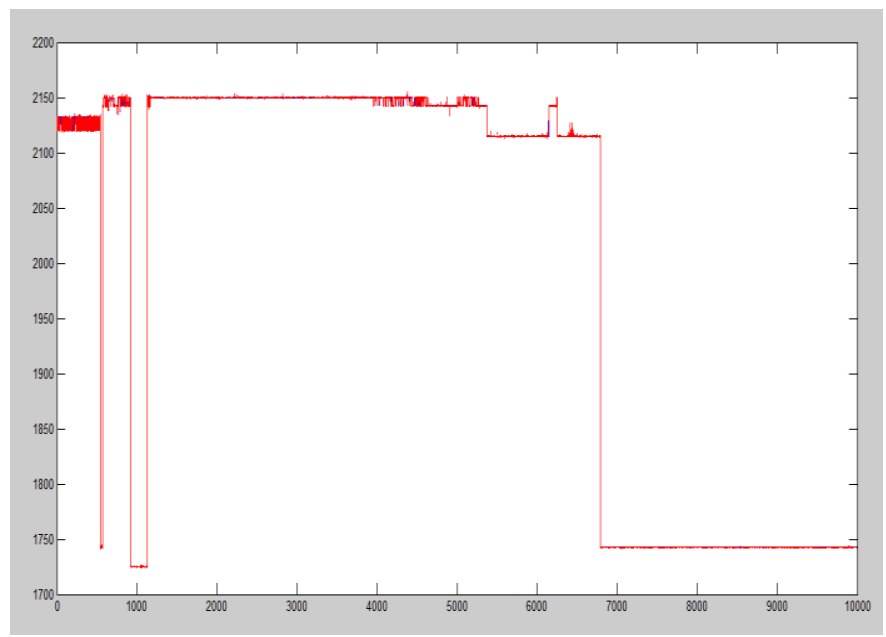

Fig. 16. Predicted and targeted values plot Radial basis NN in AWS band.

c) Analysis of Available channels frequencies using Radial Basis Neural Network: For prediction of Frequencies using Radial basis Neural Network model, same model is used as shown in Fig. 8. The best validation performance achieved in Radial Basis Neural Network is 0.7916260 as shown in Fig. 15 and 16 represent the difference between targeted values and predicted values.

\section{Comparative Analysis}

The comparison between achieved MSE founds in three different Neural Networks in cellular 850MHZ and AWS (Advance Wireless Service) 1700/2100 MHZ bands for prediction of available frequencies band are presented in the Table II and Fig. 17.

TABLE II. COMPARISION BETWEEN ACHIEVED MSE

\begin{tabular}{|l|l|l|l|}
\hline $\begin{array}{l}\text { Ann } \\
\text { Techniques }\end{array}$ & Feed Forward & Elman & Radial Basis \\
\hline $\begin{array}{l}\text { For Cellular } \\
\text { band }\end{array}$ & 0.06366 & 0.45293 & 0.09722 \\
\hline For AWS band & 0.2186 & 0.41012 & 0.79162 \\
\hline
\end{tabular}




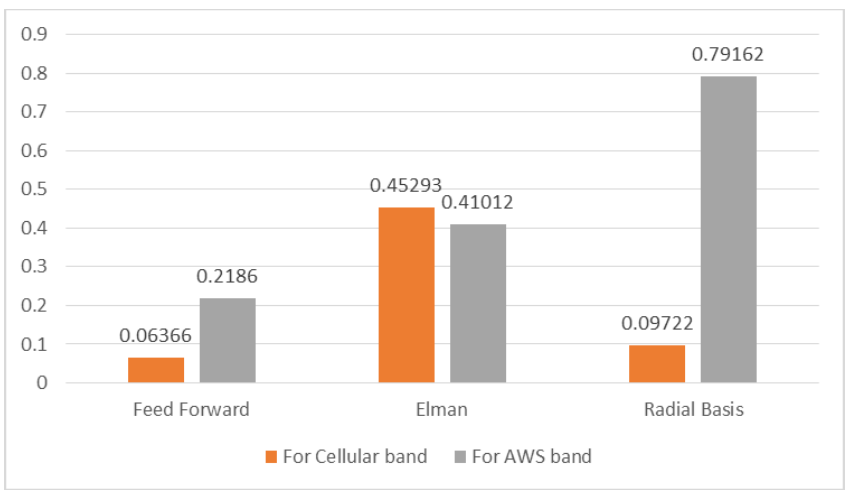

Fig. 17. Comparison between the Feed Forward NN, Elman NN and Radial Basis achieved MSE values for different Frequencies bands.

For prediction of available frequencies in cellular band and AWS band, to perform the learning in cognitive radio, feed forward neural network is most suitable as compared to other networks while Elman network are not accurate enough is the least in cellular band but performs average in AWS band and Radial basis network performs average for cellular band and least for AWS band. But the network convergence time of radial networks is much higher as compared to feed forward networks especially for big data set and cognitive radio decision must be fast and accurate also. For fast decision the feed forward network is better choice.

If we contrasted our work and other related works according to achieved MSE as in [16] author's uses feed forward networks and its MSE value is 0.27317 for different multiple factors where as in our network we achieved minimum 0.05685 MSE for feed forward networks and maximum 0.2186 for particular bands. As in [18] Multilayer feed forward performs as low as 0.06666 as comparison our feed forward network performs better according to provided conditions as multilayer increases the complexity in networks. In [18] RBF networks performs as low as 0.0835026 where as our RBF performs as 0.08722. In [20] feed forward neural networks performs as low as 0.0964 and RBF 0.0416 for predicting power. So we can say that, results can be varies according to the different parameters, algorithms and scenarios. The quantity of dataset may also varies the performance of overall network.

\section{CONCLUSION}

The MSE value as low as indicates that there is strong relationship between factors and machine learning technique. Decision of Resource Allocation should very fast and highly accurate. It can be only done by selecting a proper learning technique and ANN shows that it has best prediction accuracy in cognitive scenario. By selecting a correct neural network for required factor can increased the system reliability. It Reduced Sensing time and Interference between Primary \& Secondary users. Increases QoS as well as System accuracy and also mitigate interference between secondary user and primary user. We have implemented this method in Cellular and wireless band due to high spectrum scarcity problems in these band. This is very important as its enable the choice of the most relevant factors for training and prediction.

\section{FUTURE WORK}

The proposed method can be implement in the Broadcast band such as Low Frequency band, Medium Frequency band, High Frequency bans, Very High Frequency band, ultra-very High frequency band and TV whites space bands which are not using currently in a specific some regions. The analysis can also be performed with other different transmitting and environmental factors. Different machine learning techniques can also be implemented and consider with the similar or different datasets to improve the network performance.

\section{REFERENCES}

[1] Zhang, N., \& Mark, J. W. , “ Security-Aware Cooperation In Cognitive Radio Networks". New York: Springer, 2014.

[2] D. Ouattara, F. Krief, M. A. Chalouf, And O. Hamdi., " Spectrum Sensing Improvement in Cognitive Radio Networks for Real-Time Patients Monitoring" . Berlin, Heidelberg: Springer, pp. 179-188, 2012.

[3] Nie, N., \& Comaniciu, C., "Adaptive Channel Allocation Spectrum Etiquette for Cognitive Radio Networks", Mobile Networks and Applications, 11(6), 779-797, 2006.

[4] Ji, Z.,\&Liu, K., "Cognitive Radios Fordynamic Spectrum Access Dynamic Spectrum Sharing: A Game Theoretical Overview", IEEE Communications Magazine, 45(5), 88-94, May , 2007.

[5] Suris, J., Dasilva, L., Han, Z., \& Mackenzie, A. "Cooperative Game Theory for Distributed Spectrum Sharing", IEEE International Conference on Communications (ICC 2007), Glasgow. UK, (p. 52825287), June, 2007.

[6] Akbar, I., \& Tranter, W., "Dynamic spectrum allocation in cognitive radio using hidden Markov models: Poisson distributed case", Proceedings of the IEEE Southeast Conference, Richmond, USA, (p. 196-201), March, 2007.

[7] Ahmadi, H., Chew, Y.H., Tang, P. K., \& Nijsure, Y. , "Predictive opportunistic spectrum access using learning based hidden Markov models", 22nd IEEE International Symposium on Personal Indoor and Mobile Radio Communications (PIMRC 2011), Toronto, Canada, (p. 401- 405), September, 2011.

[8] Ahmadi, H.,Macaluso, I., \& Dasilva, L. (2013, June). "The effect of the spectrum opportunities diversity on opportunistic access", IEEE International Conference on Communications (ICC 2013), Budapest, Hungary, (p. 2829-2834), June, 2013.

[9] Macaluso, I., Ahmadi, H., \& Dasilva, L., "Fungible Orthogonal Channel Sets for Multi-User Exploitation of Spectrum", IEEE Transactions on Wireless Communications, 14(4), (p.2281-2293), April, 2015.

[10] Lan, K., Luo, M., Long, C., Zhang, J., \& Zhao, H., "A Spectrum Prediction Approach based on Neural Networks Optimized by Genetic Algorithm in Cognitive Radio Networks", 10th International Conference on Wireless Communications, Networking and Mobile Computing WiCOM, September, 2014.

[11] Elhachmi, J., \& Guennoun, Z., "Cognitive radio spectrum allocation using genetic algorithm", EURASIP Journal on Wireless Communications and Networking, 2016.

[12] Zhao, J., Li, F., \& Zhang, X., "Parameter adjustment based on improved genetic algorithm for cognitive radio networks", The Journal of China Universities of Posts and Telecommunications, 19(3),(p. 22-26), 2012.

[13] Sun, A., Liang, T., Zhang, Y., \& Lu, W., "An Adaptive Genetic Based Cognitive Radio Parameter Adjustment Algorithm", Seventh International Symposium on Computational Intelligence and Design, 2014.

[14] HAYKIN, S., “ Neural Networks: A Comprehensive Foundation”, (2nd ed.), Prenticel Hall, 1998.

[15] Baldo, N., Tamma, B.,Manoj, B., Rao, R., \& Zorzi, M., "A Neural Network Based Cognitive Controller for Dynamic Channel Selection", IEEE International Conference on Communications (ICC 2009), Dresden, Germany, (p. 1-5), June, 2009.

[16] Singh, R., \& Kansal, S., "Artificial neural network based spectrum recognition in cognitive radio", IEEE Students' Conference on Electrical, Electronics and Computer Science, (SCEECS), 2016. 
[17] Agarwal, A., Dubey, S., Khan, M. A., Gangopadhyay, R., \& Debnath, S., "Learning based primary user activity prediction in cognitive radio networks for efficient dynamic spectrum access", 2016 International Conference on Signal Processing and Communications, (SPCOM), 2016.

[18] Winston, O., Thomas, A., \& Okelloodongo, W., "Comparing performance of MLP and RBF neural networks for TV idle channel prediction in Cognitive Radio", 2013 Pan African International Conference on Information Science, Computing and Telecommunications, (PACT), 2013.

[19] Y Yang, H Jiang, C Liu, Z Lan., "Research on cognitive radio engine based on genetic algorithm and radial basis function neural network", Proceedings of the Spring Congress on Engineering and Technology, 2012.
[20] Iliya, S., Goodyer, E., Gow, J., Shell, J., \& Gongora, M., "Application of Artificial Neural Network and Support Vector Regression in Cognitive Radio Networks for RF Power Prediction Using Compact Differential Evolution Algorithm", Proceedings of the 2015 Federated Conference on Computer Science and Information Systems, 2015.

[21] Gatla, V., Kulkarni, A., \& Venkatesan, M., "Feed forward neural network based learning scheme for cognitive radio systems", Third International Conference on Computational Intelligence and Information Technology, CIIT, 2013.

[22] Tan, X., Huang, H., \& Ma, L., "Frequency allocation with Artificial Neural Networks in cognitive radio system", IEEE Tencon - Spring, 2013.

[23] Retrieved from:https://sms-sgs.ic.gc.ca/eic/site/sms-sgsprod.nsf/eng/ h_00010.html. 